\title{
Population Pharmacokinetic Modelling of Ceftazidime and Avibactam in the Plasma and Epithelial Lining Fluid of Healthy Volunteers
}

\author{
Richard Dimelow $^{1,4} \cdot$ James G. Wright $^{1} \cdot$ Merran MacPherson $^{1,5} \cdot$ Paul Newell $^{2,6} \cdot$ Shampa Das $^{2,3}$ (D)
}

Published online: 27 July 2018

(c) The Author(s) 2018

\begin{abstract}
Objectives Our objective was to develop population pharmacokinetic (PK) models for ceftazidime and avibactam in the plasma and epithelial lining fluid (ELF) of healthy volunteers and to compare ELF concentrations to plasma PK/pharmacodynamic (PD) targets.

Methods Plasma and ELF population PK models were developed for ceftazidime and avibactam concentration data from 42 subjects (NCT01395420). Two- and three-compartment plasma PK models were fitted to ceftazidime and avibactam plasma PK data, and different plasma-ELF linked models were evaluated. Using best-fitting models, plasma and ELF concentration-time profiles were simulated for 1000 subjects. ELF concentration-time profiles for ceftazidime/avibactam 2000-500 mg every $8 \mathrm{~h}$ were compared with plasma PK/PD targets for ceftazidime (50\% of time above $[f \mathrm{~T}>] 8 \mathrm{mg} / \mathrm{l})$ and avibactam $(50 \% f \mathrm{~T}>1 \mathrm{mg} / \mathrm{l})$.

Results Three-compartment PK models best fitted the plasma concentration data for ceftazidime and avibactam. ELF data for both drugs were best described by a direct response (instantaneous equilibrium) model. Ceftazidime plasma-ELF relationships were best described by a saturable Michaelis-Menten model. For avibactam, departure from plasma-ELF relationship linearity was more modest than for ceftazidime. ELF:plasma penetration ratios of both ceftazidime (52\%) and avibactam $(42 \%)$ at plasma concentrations relevant for efficacy $(\sim 8 \mathrm{mg} / \mathrm{l}$ for ceftazidime and $\sim 1 \mathrm{mg} / \mathrm{l}$ for avibactam) were greater than previously calculated using non-compartmental area under the curve (AUC) methods, which average across the entire concentration range. Ceftazidime and avibactam ELF exposures exceeded their respective plasma PK/PD time-above-threshold targets by the dosing interval mid-point in most subjects.
\end{abstract}

Conclusions This compartmental modelling analysis suggests ELF exposures of both ceftazidime and avibactam exceed levels required for efficacy in plasma.

Shampa Das

shampa.das@liverpool.ac.uk

1 Wright Dose Ltd, Altrincham, Cheshire, UK

2 AstraZeneca Global Medicines Development, Alderley Park, Macclesfield, Cheshire, UK

3 Present Address: Antimicrobial Pharmacodynamics and Therapeutics, Department of Molecular and Clinical Pharmacology, University of Liverpool, Sherrington Building, Liverpool L69 3GA, UK

4 Present Address: GlaxoSmithKline, Stevenage, UK

5 Present Address: SGS Exprimo, Mechelen, Belgium

6 Present Address: Knutsford, Cheshire, UK 


\section{Key Points}

Population pharmacokinetic (PK) models were developed to describe the relationship of ceftazidime and avibactam concentrations in plasma with those in epithelial lining fluid (ELF) in healthy volunteers and to compare ELF concentrations with plasma $\mathrm{PK} /$ pharmacodynamic (PD) targets.

The analysis used data from the phase I study which, using non-compartmental calculations, showed that the area under the curve (AUC) of each drug in the ELF is approximately $31-35 \%$ of that in plasma. However, non-compartmental AUC methods average across the studied concentration range and therefore do not necessarily calculate penetration at the most clinically relevant concentrations.

In the current compartmental modelling analysis, ELF penetration of both ceftazidime (52\%) and avibactam (42\%) was greater than previously calculated at plasma concentrations relevant for efficacy ( $~ 8 \mathrm{mg} / \mathrm{l}$ for ceftazidime and $\sim 1 \mathrm{mg} / \mathrm{l}$ for avibactam), and results suggest ELF exposures of both drugs exceeded levels required for efficacy in plasma.

\section{Introduction}

Antibiotic efficacy in pneumonia requires sufficient unbound drug concentrations at the pulmonary site of infection, which in turn depends on factors such as penetration into lung tissue, the lack of antibiotic inactivation by pulmonary surfactant, the degree of plasma protein binding and the clearance rate of the antibiotic [1-4]. Lack of consideration of these factors is thought to have caused the failure of some antibiotic phase III trials in nosocomial pneumonia (NP)/ ventilator-associated pneumonia (VAP) [5]. Determination of the bronchopulmonary availability of antibiotics in epithelial lining fluid (ELF) allows penetration into the lung to be characterized [6]. Understanding the relationships between plasma and pulmonary tissue antibiotic pharmacokinetics (PK) is therefore important to ensure that treatments intended for patients with NP/VAP can achieve clinically relevant exposures at the infection site.

Ceftazidime/avibactam has recently been approved in Europe and the USA for the treatment of adults with complicated intra-abdominal infections (in combination with metronidazole) and urinary tract infections, including acute pyelonephritis $[7,8]$. Ceftazidime/avibactam has also been granted approval in Europe for the treatment of hospital-acquired pneumonia/VAP [7] and in the USA for hospital-acquired bacterial pneumonia and ventilator-associated bacterial pneumonia [8].

For most bacterial infections, achievement of sufficient plasma exposure according to defined PK/pharmacodynamic (PD) parameters is considered to relate to clinical efficacy [9], and this approach has been used to guide dosage selection and determination of susceptibility and resistance breakpoints for antimicrobial therapies. Plasma PK/ PD targets for ceftazidime and avibactam were derived from preclinical (mouse) data: $(50 \% f \mathrm{~T}>8 \mathrm{mg} / \mathrm{l}$ for ceftazidime and $50 \% f \mathrm{~T}>1 \mathrm{mg} / \mathrm{l}$ for avibactam) [10-12]. Ceftazidime and avibactam demonstrate higher penetration in ELF in human models than in mouse models, supporting the use of plasma as a surrogate endpoint for ELF exposures [13]. The plasma targets were used in population PK modelling analyses $[14,15]$ to guide selection of ceftazidime/avibactam dosage regimens for the ceftazidime/avibactam phase III clinical trials, including the REPROVE trial in patients with NP/VAP. However, plasma-derived targets are likely to overestimate exposures required for efficacy in the ELF [13], as protein levels in ELF are lower than those in plasma [2] and therefore the free fraction in ELF is likely to be higher than in plasma.

The objective of this analysis was to develop population PK models for ceftazidime and avibactam in the plasma and ELF using subject PK data from a phase I study of ceftazidime/avibactam penetration into ELF [16] to provide further understanding of ELF penetration in humans. Previous analyses of the study data have used non-compartmental area under the curve (AUC) calculations to characterize ceftazidime and avibactam plasma-ELF relationships. However, non-compartmental AUC methods average across the studied concentration range and hence do not necessarily calculate penetration at the most clinically relevant concentrations. An improved understanding of the non-linearity in plasma-ELF relationships enabled by a compartmental modelling approach may facilitate dose optimization for future antibiotics and combinations.

\section{Methods}

\subsection{Phase I Epithelial Lining Fluid (ELF) Study}

A previously reported phase I open-label study in healthy volunteers (NCT01395420) assessed ceftazidime and avibactam steady-state concentrations in plasma and ELF based on AUC values from non-compartmental analysis [16]. The study enrolled 43 healthy male volunteers to receive ceftazidime $2000 \mathrm{mg}+$ avibactam $500 \mathrm{mg}$ (cohort A; $n=22$ ) or ceftazidime $3000 \mathrm{mg}$ + avibactam $1000 \mathrm{mg}$ (cohort B; $n=21$ ) by $2 \mathrm{~h}$ intravenous infusion, every $8 \mathrm{~h}$ ( $\mathrm{q} 8 \mathrm{~h}$ ) for 3 days (total 
of nine doses per subject). PK data were available for a total of 42 subjects ( 22 in cohort $\mathrm{A}$ and 20 in cohort B).

\subsection{Model Development}

Using data from these 42 subjects, population PK models for plasma and ELF were developed separately for ceftazidime and avibactam using the sequential iterative two-stage (ITS) and importance sampling (IMP) expectation-maximization methods in NONMEM version 7.2.0. For each drug, a twocompartment and a three-compartment PK model with firstorder elimination, parameterized by clearance and volume parameters and coded as a series of differential equations, were fitted to the plasma concentration-time data and compared. The model that best described the data was selected, and a population ELF PK model driven by the predicted plasma concentration was subsequently developed. Postprocessing of the final models and subsequent simulations were carried out in $\mathrm{R}$ version 2.15.0.

The joint plasma/ELF PK model was fitted simultaneously to the plasma and ELF concentration data, but only the parameters of the ELF PK model were allowed to vary, the fixed- and random-effect parameters of the plasma PK model being held fixed at their values previously estimated from fitting to the plasma concentration data. Several different direct response and effect site equilibrium models were then fitted to the ELF PK data to evaluate the presence of delayed equilibration, and the model that offered the best fit to the data was selected as the final model. Different plasma-ELF link functions (proportional, power and saturable) were investigated for both model types. Residual noise in plasma concentration was modelled as proportional to the predicted plasma concentration. With one ELF observation per individual, residual noise in the ELF concentrations could not be independently estimated from the data and was therefore fixed to the median value for proportional noise from the plasma population PK model. Between-subject variability in parameters was modelled as being log-normally distributed.

\subsubsection{Direct Response Model}

A direct response model assumes that drug concentration in ELF is in an instantaneous equilibrium with the plasma concentration based on rapid drug exchange between plasma and ELF. ELF concentration is therefore a function of plasma concentration, i.e. $C_{\mathrm{ELF}}(t)=f\left[C_{\mathrm{p}}(t)\right]$.

2.2.1.1 Plasma-ELF Link Functions Three different forms were considered for this function. The simplest was a proportional model $C_{\mathrm{ELF}}(t)=\mathrm{EPR} \cdot C_{\mathrm{p}}(t)$, where EPR is the ELF/plasma penetration ratio. Second, a power model, $C_{\mathrm{ELF}}(t)=\operatorname{EPR}\left(\frac{1 \mathrm{mg}}{L}\right) \cdot\left[C_{\mathrm{p}}(t)\right]^{\mathrm{POW}}$, where EPR $(1 \mathrm{mg} / \mathrm{l})$ is the ELF/plasma penetration ratio at a plasma concentration of $1 \mathrm{mg} / \mathrm{l}$, and the POW parameter allows the penetration ratio to change with plasma concentration. For POW $=1$, the model is identical to the proportional model, whereas for values $<1$, the penetration ratio decreases as concentration increases. Finally, a saturable $E_{\mathrm{MAX}}$ model was considered $C_{\mathrm{ELF}}(t)=\frac{E_{\mathrm{MAX}} \cdot C_{\mathrm{p}}(t)}{K_{\mathrm{M}}+C_{\mathrm{p}}(t)}$, where $E_{\mathrm{MAX}}$ is the maximum ELF concentration achievable, and $K_{\mathrm{M}}$ is the plasma concentration at which half of $E_{\mathrm{MAX}}$ is achieved.

\subsubsection{Effect Site Equilibration Model}

The possibility of a delay in ELF concentrations relative to plasma concentrations was also evaluated using an effect site equilibrium model, where ELF concentration, $C_{\mathrm{ELF}}(t)$, is represented by a model compartment, the rate of transfer into the ELF compartment is defined as a function of plasma concentration, $f\left[C_{\mathrm{p}}(t)\right]$ i.e. $\frac{\mathrm{d} C_{\mathrm{ELF}}(t)}{\mathrm{d} t}=K_{\mathrm{ELF}} \cdot f\left[C_{\mathrm{p}}(t)\right]-K_{\mathrm{ELF}} \cdot C_{\mathrm{ELF}}(t)$, and the rate constant $K_{\mathrm{ELF}}$ determines the ELF compartment half-life, i.e. $\tau=\frac{\ln 2}{K_{\mathrm{ELF}}}$.

\subsection{Model Selection}

Model selection was primarily based on the objective function value (OFV) provided by NONMEM ( -2 multiplied by the logarithm of the likelihood) and visual inspection of graphical diagnostics. For a more complicated model to be selected, it had to provide a significant improvement in fit over the contending model $(p<0.05, \Delta \mathrm{OFV}$ at least -3.84 with 1 degree of freedom for nested models).

\subsection{Model Evaluation}

The adequacy of the models was evaluated using a simulation-based visual predictive check (VPC) method. The models were used to simulate 1000 replicates of the analysis dataset with NONMEM. The simulated predictions at the 5th, 50th and 95th percentiles were then superimposed on the observed plasma and ELF concentrations. If the observed 5th, 50th and 95th percentiles were in agreement with the corresponding simulated percentiles, then the range of concentrations over the population as predicted by the model was typical of the spread observed in the data, and the model could be considered a reliable representation of the population. Using the final models, ELF penetration ratios were calculated for ceftazidime and avibactam with respect to their total concentrations in plasma and ELF. 


\subsection{Model Simulations}

Simulations of 1000 subjects receiving ceftazidime/avibactam 2000-500 mg q8 $\mathrm{h}$ were performed in NONMEM using the final avibactam and ceftazidime models. The relationships between plasma and ELF (total) concentrations were characterized by comparing the 5 th, 50th and 95th simulated percentiles with the observed data on plots of ELF versus plasma concentrations and ELF concentrations versus time. Predicted and observed ELF concentrations were compared with plasma PK/PD targets for ceftazidime $(50 \% f \mathrm{~T}>8 \mathrm{mg} / \mathrm{l})$ and avibactam $(50 \% f \mathrm{~T}>1 \mathrm{mg} / \mathrm{l})$, derived from preclinical experiments [10-12]. For ceftazidime, 50\% $f \mathrm{~T}>$ minimum inhibitory concentration (MIC) is well-established as the PK/PD target that best correlates with efficacy [17-20]. The $50 \% f \mathrm{~T}>8 \mathrm{mg} / \mathrm{l}$ target used here is supported by global surveillance data showing that a ceftazidime-avibactam MIC $\leq 8 \mathrm{mg} / \mathrm{l}$ includes $\geq 90 \%$ of clinical isolates of Enterobacteriaceae and Pseudomonas aeruginosa [21-24], and $\leq 8 \mathrm{mg} / \mathrm{l}$ has been determined as the "susceptible" MIC breakpoint for ceftazidime-avibactam against these bacteria $[7,8,25]$. The $50 \% f \mathrm{~T}>8 \mathrm{mg} / \mathrm{l}$ ceftazidime target thus represents the upper end of exposure required for clinical efficacy and was therefore considered appropriate to provide a conservative estimate of the clinical relevance of ELF exposures.

\section{Results}

\subsection{Plasma Pharmacokinetic (PK) Models}

Between-subject variability was adequately characterized using a full covariance matrix, and residual variability was modelled using the proportional residual model. For both ceftazidime and avibactam, the OFV drop was significant (195 and 272 units, respectively) for the addition of two extra parameters for the three-compartment model $\left(V_{3}\right.$ and $Q_{13}$ ), indicating that ceftazidime and avibactam plasma PK for this dataset were both best described by three-compartment kinetics. The two-compartment model also produced a reasonable fit to the data, but the more complex model was selected to maximize the accuracy of the fit to the plasma PK data, for use as an input to the ELF PK model. Ceftazidime and avibactam final plasma PK model parameters are shown in Table 1.

\subsection{Ceftazidime ELF PK Model}

Three different plasma-ELF link functions (proportional, power and saturable) were evaluated in the direct-response ELF PK model. A direct-response model with a saturable plasma-ELF link function had a significantly lower OFV than the other two plasma-ELF link functions and provided the best fit to the data. The saturable plasma-ELF link function was 35 units lower than the proportional link function and eight units lower than the power link function.
Table 1 Final plasma population pharmacokinetic model parameters for ceftazidime and avibactam

\begin{tabular}{llllll}
\hline Drug & Parameter & Median $(\%)$ & \multicolumn{2}{l}{ Between-subject variability } \\
\cline { 4 - 5 } & & & 5 th percentile & 95 th percentile & CV \% \\
\hline Ceftazidime & $V_{1}(1)$ & $10.32 \pm 8$ & 7.73 & 13.78 & 18 \\
& $V_{2}(1)$ & $5.82 \pm 9$ & 4.37 & 7.75 & 18 \\
& $V_{3}(\mathrm{l})$ & $0.64 \pm 8$ & 0.43 & 0.95 & 25 \\
& $Q_{12}(1 / \mathrm{h})$ & $6.87 \pm 24$ & 2.54 & 18.55 & 66 \\
& $Q_{13}(\mathrm{l} / \mathrm{h})$ & $0.040 \pm 33$ & 0.016 & 0.101 & 61 \\
& $\mathrm{CL}(\mathrm{l} / \mathrm{h})$ & $6.55 \pm 2$ & 5.59 & 7.68 & 10 \\
& $\mathrm{RESM}$ & $0.101 \pm 9$ & 0.044 & 0.233 & 54 \\
& $V_{1}(\mathrm{l})$ & $15.10 \pm 10$ & 13.08 & 17.44 & 9 \\
& $V_{2}(\mathrm{l})$ & $6.52 \pm 15$ & 4.32 & 9.84 & 25 \\
& $V_{3}(\mathrm{l})$ & $1.58 \pm 5$ & 1.15 & 2.17 & 60 \\
& $Q_{12}(1 / \mathrm{h})$ & $5.43 \pm 42$ & 2.19 & 13.45 & 75 \\
& $Q_{13}(1 / \mathrm{h})$ & $0.14 \pm 41$ & 0.05 & 0.43 & 7 \\
& $\mathrm{CL}(\mathrm{l} / \mathrm{h})$ & $12.5 \pm 1$ & 11.1 & 0.239 & 45 \\
\hline
\end{tabular}

$C L$ clearance, $C V$ coefficient of variation, $h$ hour, $l$ litre, $Q_{12}$ flow rate to compartment $2, Q_{13}$ flow rate to compartment 3, RESMp residual noise (median) for plasma, $V_{l}$ volume of distribution compartment $1, V_{2}$ volume of distribution compartment $2, V_{3}$ volume of distribution compartment 3 
The effect-site equilibrium model did not lower the OFV relative to the equivalent direct-response model. Furthermore, the estimate of the ELF concentration rate constant $\left(K_{\mathrm{ELF}}\right)$ in the effect-site equilibrium model also indicated that the ELF concentration half-life was short (13 $\mathrm{min}$ ), and so the equilibration between plasma and ELF was rapid. The ceftazidime ELF data were therefore best described by a direct-response model (i.e. instantaneous equilibrium), and the three-compartment model coupled with the direct saturable-response model was therefore selected to best characterize the response and between-subject variability in the ceftazidime ELF concentration-time course profiles.

Final ELF PK model parameters for ceftazidime are shown in Table 2. The maximum possible ELF concentration $\left(E_{\mathrm{MAX}}\right)$ was predicted to be $45.4 \mathrm{mg} / \mathrm{l}$; half this ELF concentration $(22.7 \mathrm{mg} / \mathrm{l})$ would be realised for a plasma concentration $\left(K_{\mathrm{M}}\right)$ of $71.7 \mathrm{mg} / \mathrm{l}$. The maximum plasma drug concentration $\left(C_{\max }\right)$ for a standard dose of ceftazidime $2000 \mathrm{mg}$ is approximately $70 \mathrm{mg} / \mathrm{l}$ [26]. At $70 \mathrm{mg} / \mathrm{l}$, ceftazidime ELF concentration $\left(\frac{E_{\mathrm{MAX}} \cdot C_{\mathrm{p}}(t)}{K_{\mathrm{M}}+C_{\mathrm{p}}(t)}\right)$ was $22.5 \mathrm{mg} / \mathrm{l}$, corresponding to a penetration ratio of $32.1 \%$. As the plasma concentration approached zero, the ratio of the ELF to plasma concentration $\left(E_{\mathrm{MAX}} / K_{\mathrm{M}}\right)$ was $63.3 \%$. An ELF ceftazidime concentration relevant for efficacy of $\sim 8 \mathrm{mg} / \mathrm{l}$ was achieved at a plasma concentration of $15.3 \mathrm{mg} / \mathrm{l}$, corresponding to an ELF penetration ratio of $52 \%$.

\subsection{Avibactam ELF PK Model}

The three-compartment avibactam plasma PK model was coupled with the three types of plasma-ELF link functions in a direct-response model, and-in the same way as for ceftazidime-fitted to the combined avibactam plasma and ELF data. A power model plasma-ELF link function resulted in the lowest OFV and provided the best fit to the data. The power model plasma-ELF link function was $22 \mathrm{OFV}$ units lower than the proportional link function and six units lower than the saturable link function.
As with ceftazidime, the effect-site equilibrium model did not lower the OFV relative to the equivalent direct-response model. The equilibration between plasma and ELF was also estimated to be rapid for avibactam, as the estimated $K_{\mathrm{ELF}}$ of the effect-site equilibrium model indicated that the avibactam ELF concentration half-life was short (8 min). As with ceftazidime, the avibactam ELF data were therefore also best described by a direct-response model. The three-compartment plasma PK model coupled with the direct-response power model was therefore selected as the final model to best characterize the response and between-subject variability in the avibactam ELF concentration-time profiles.

Final ELF PK model parameters for avibactam are shown in Table 2. At plasma avibactam concentrations relevant for efficacy ( 1 mg/l), penetration into ELF was $47 \%$. An ELF concentration relevant for efficacy of $1 \mathrm{mg} / \mathrm{l}$ avibactam was achieved at a median plasma concentration of $2.4 \mathrm{mg} / \mathrm{l}$, corresponding to $42 \%$ penetration. The $C_{\max }$ for a standard avibactam dose of $500 \mathrm{mg}$ is approximately $12 \mathrm{mg} / \mathrm{l}$ [26]. At $12 \mathrm{mg} / \mathrm{l}$, ELF concentration (EPR・Cp^POW) was $4.0 \mathrm{mg} / \mathrm{l}$, corresponding to a decreased ratio of ELF to plasma concentration of $33.2 \%$.

\subsection{Visual Predictive Checks}

Prediction-corrected VPCs for the final joint models of ceftazidime and avibactam PK in plasma and ELF are shown in Fig. 1. In all cases, there was good agreement between the simulated (shaded regions) and observed (solid lines) percentiles, indicating that the models are likely to be an accurate and reliable representation of the study population.

\subsection{Model Simulations}

Simulated ceftazidime and avibactam ELF versus plasma concentrations for each subject are shown in Fig. 2. Saturability in the ELF ceftazidime concentrations was observed at higher plasma ceftazidime concentrations $(>100 \mathrm{mg} / \mathrm{l})$, exceeding the median $C_{\max }$ predicted in plasma. In contrast, there was only a slight departure from linearity in ELF and
Table 2 Final epithelial lining fluid population pharmacokinetic model parameters for ceftazidime and avibactam

\begin{tabular}{llllll}
\hline Drug & Parameter & Median & 5th percentile & 95th percentile & CV \% \\
\hline Ceftazidime & $E_{\mathrm{MAX}}, \mathrm{mg} / \mathrm{l}$ & $45.4 \pm 12 \%$ & 30.9 & 66.5 & 24 \\
& $K_{\mathrm{M}}, \mathrm{mg} / \mathrm{l}$ & $71.7 \pm 22 \%$ & 18.8 & 273.3 & 97 \\
& RESM & 0.101 & 0 & 0 & 0 \\
Avibactam & EPR $(1 \mathrm{mg} / \mathrm{l})$ & $0.472 \pm 11 \%$ & 0.172 & 1.298 & 68 \\
& POW & $0.860 \pm 5 \%$ & 0.588 & 1.259 & 23 \\
& RESM $_{\mathrm{ELF}}$ & 0.117 & 0 & 0 & 0 \\
\hline
\end{tabular}

$C V$ coefficient of variation, ELF epithelial lining fluid, EPR $(1 \mathrm{mg} / \mathrm{l})$ ELF penetration ratio at plasma concentration of $1 \mathrm{mg} / \mathrm{l}, E_{M A X}$ maximal concentration, $K_{M}$ Michaelis-Menten constant, $P O W$ power term, $R E S$ $M_{E L F}$ residual noise (median) for ELF, $K_{E L F}$ rate constant for ELF 

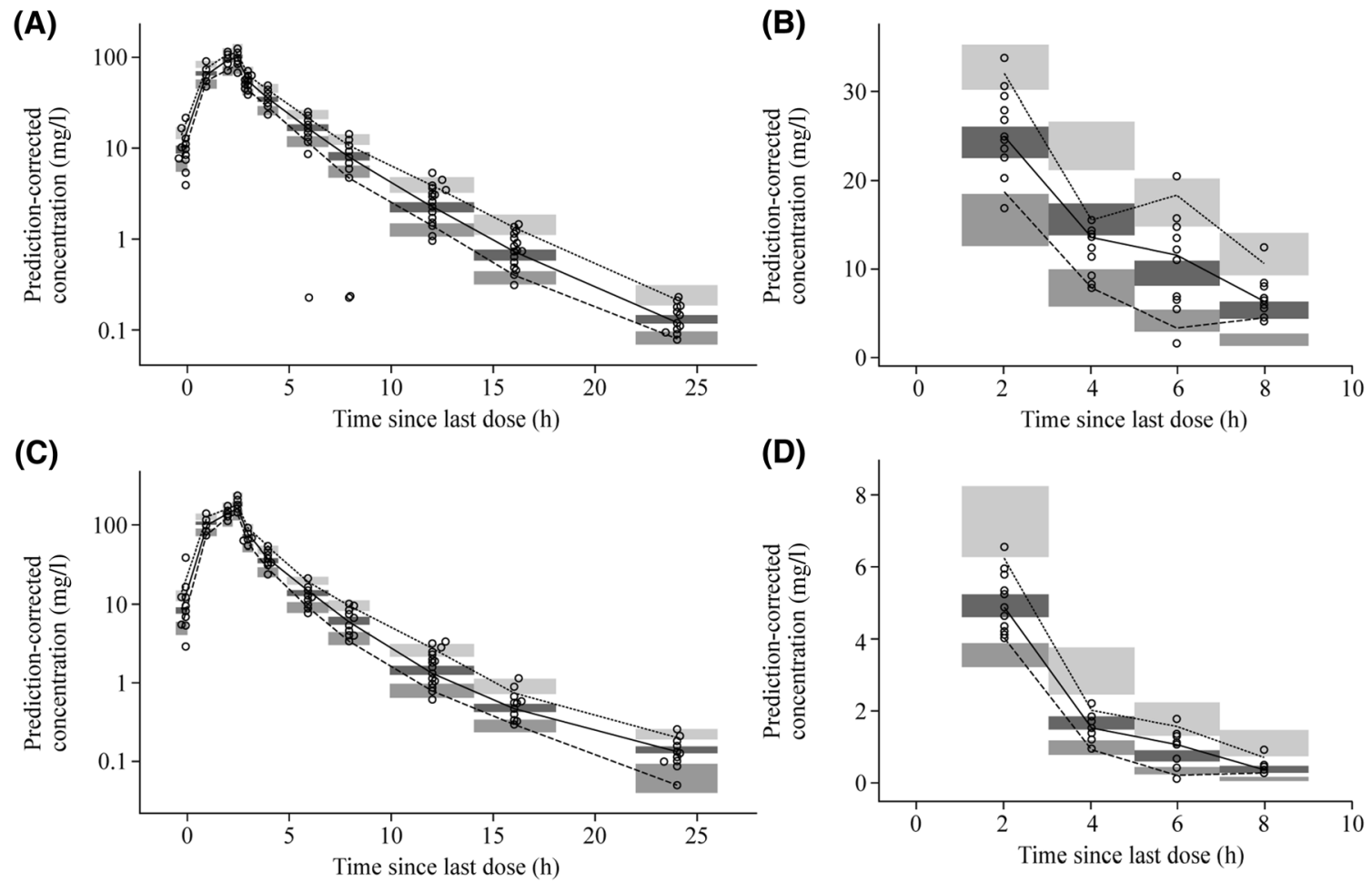

Fig. 1 Prediction-corrected visual predictive checks versus time from start of infusion of the last dose for a final ceftazidime plasma population pharmacokinetic model, b final ceftazidime epithelial lining fluid pharmacokinetic model, $\mathbf{c}$ final avibactam plasma pharmacokinetic model, and $\mathbf{d}$ final avibactam epithelial lining fluid phar- macokinetic model. The solid lines represent median, 5th and 95th percentiles of observations. Shaded dark, mid- and light grey regions encompass $90 \%$ of simulated $(n=1000)$ values of predicted medians, 5 th, and 95th percentiles, respectively. Circles represent predictioncorrected values of observed data plasma avibactam concentrations, observed at plasma concentrations $>20 \mathrm{mg} / \mathrm{l}$. In Fig. 3, simulated ELF concentration-time profiles are overlaid with ceftazidime and avibactam plasma PK/PD targets $(8 \mathrm{mg} / \mathrm{l}$ for ceftazidime and $1 \mathrm{mg} / \mathrm{l}$ for avibactam). At the midpoint of the 8 -h dosing interval (i.e. 4 h post dose), median ( 5 th to 95 th percentile) simulated ELF ceftazidime and avibactam concentrations were 14.7 (7.1-23.4) and $1.4(0.75-2.85) \mathrm{mg} / \mathrm{l}$, respectively. Although the targets are derived for plasma and are therefore likely to overestimate the concentrations required for efficacy in target tissues, most subjects still achieved ceftazidime and avibactam ELF exposures exceeding their respective plasma PK/PD targets beyond the midpoint of the dosing interval.

\section{Discussion}

We developed population PK models to describe the relationship of ceftazidime and avibactam in plasma with that in the ELF of healthy volunteers and to compare ELF concentrations to plasma PK/PD targets. While studies to determine the bronchopulmonary availability of antibiotics in ELF are regularly performed, the measurement of drug concentrations in ELF is technically challenging [2], involving repeated sampling, often in critically ill patients, and results can be difficult to reproduce and interpret [2]. To reach ELF, drugs need to cross the blood-alveolar barrier, which is composed of alveolar epithelial cells that form two membranes, the capillary wall and alveolar wall, separated by interstitial fluid [2]. Molecular weight and lipophilicity are considered to determine the extent of diffusion across these membranes, and intracellular uptake is also a factor for some antibiotics; however, whether active transport plays a role for specific antibiotics is unknown [2, 27]. For $\beta$-lactams such as ceftazidime, which are relatively lipophobic, variable ELF concentrations have been reported. Avibactam is a novel (non- $\beta$-lactam) agent for which limited data on ELF penetration are currently available.

The present analyses used data from the phase I ELF study, which has previously shown, using a non-compartmental approach, that the AUC of each drug in the ELF is approximately $31-35 \%$ of that in plasma [16]. Non-compartmental methods to calculate AUC average across the concentration range sampled in the study and thus assume that ELF penetration is constant across the concentration range studied. However, it is important to accurately estimate the 


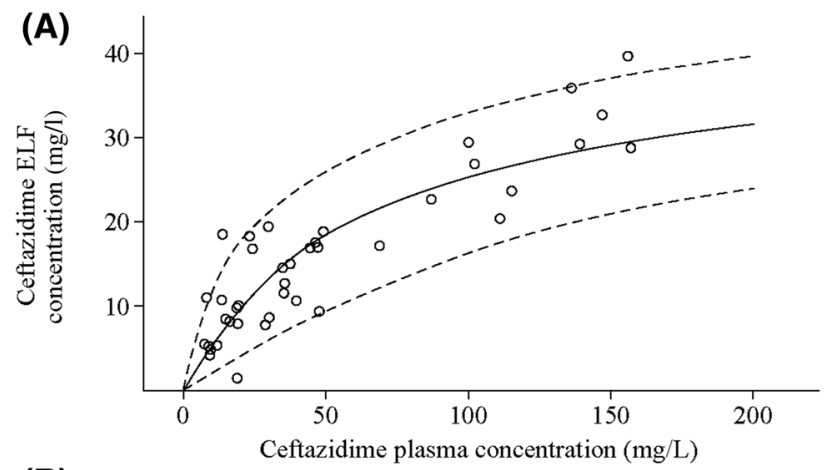

(B)

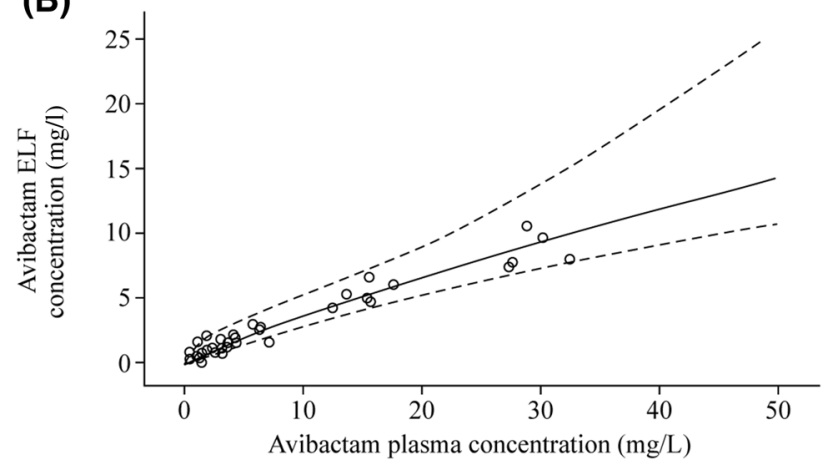

Fig. 2 Simulated epithelial lining fluid (ELF) concentrations versus plasma concentrations in 1000 subjects receiving ceftazidime/avibactam 2000-500 mg every $8 \mathrm{~h}$ for a ceftazidime and b avibactam. The solid line represents the population median percentile of 1000 simulated individuals, and the broken lines are 5th and 95th percentiles. Circles represent observed data points

degree of penetration at concentrations required for efficacy. Therefore, the present analysis applied compartmental population PK modelling to better characterize the PK of ceftazidime and avibactam in the plasma and ELF of healthy volunteers. The observed non-linearity in the ELF-plasma concentration relationships of ceftazidime and avibactam suggest that saturable systems might play a role in the penetration of these drugs across the blood-alveolar barrier.

As only a single ELF concentration was available for each subject, it was not possible to distinguish residual and between-subject variance for the ELF data. The residual variability was therefore fixed at the same value as that inferred from the plasma data, and between-subject variances in the ELF concentrations were estimated on this basis. If a higher residual noise estimate were used, the between-subject variance would decrease correspondingly. However, VPCs confirmed that the value assumed for residual noise produced reasonable simulated profiles, consistent with the data. Nonetheless, the estimates of between-subject variability are conditional on the assumed value for residual noise.

The penetration of ceftazidime and avibactam from plasma into ELF was very rapid and was accurately modelled as an instantaneous equilibrium. The possibility of delayed equilibration between ELF and plasma was investigated for

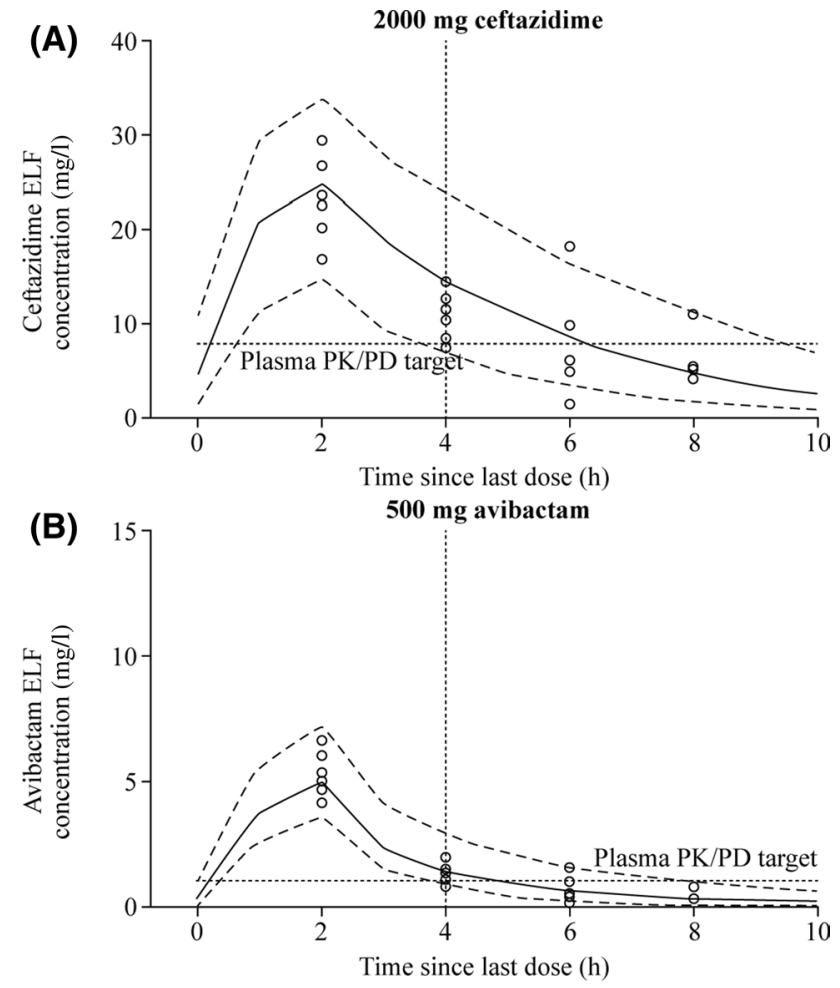

Fig. 3 Simulated total epithelial lining fluid (ELF) concentrationtime profiles in 1000 subjects receiving ceftazidime/avibactam 2000 $500 \mathrm{mg}$ every $8 \mathrm{~h}$ for $\mathbf{a}$ ceftazidime and $\mathbf{b}$ avibactam. The solid line represents the median percentile of 1000 simulated individuals, and the broken lines are 5th and 95th percentiles. The circles represent the observed data points. The horizontal hashed line represents the plasma pharmacokinetic/pharmacodynamic target for ceftazidime (50\% $f \mathrm{~T}>$ minimum inhibitory concentration of $8 \mathrm{mg} / \mathrm{l})$ and avibac$\operatorname{tam}(50 \% f \mathrm{~T}>1 \mathrm{mg} / \mathrm{l})$, and the vertical hashed line represents the midpoint of the 8-h dosing interval

both drugs but did not improve the model fit in either case. Nonetheless, short equilibration half-lives of $13 \mathrm{~min}$ for ceftazidime and 8 min for avibactam could be estimated with reasonable standard errors. Although these models did not improve on the instant equilibration in the final models, they effectively exclude the possibility of a slow equilibration. Hence, if there is any delay in equilibration, it is measured in minutes. This is consistent with the physiology of the lung, where ELF is separated from plasma by a single layer of alveolar epithelial cells with a high transporter complement.

Ceftazidime plasma-ELF relationships were best described by a saturable Michaelis-Menten model, such that ELF penetration was higher at low plasma concentrations but saturable at very high plasma concentrations, estimated as $>250 \mathrm{mg} / \mathrm{l}$. The ceftazidime ELF concentration has an estimated maximum at $45.4 \mathrm{mg} / \mathrm{l}$ (population median), with a half-maximal ELF concentration achieved at a median plasma concentration of $71.7 \mathrm{mg} / \mathrm{l}$, comparable to the typical $C_{\max }$ after administration of the standard 
dose of ceftazidime/avibactam $2000-500 \mathrm{mg}$ by 2-h intravenous infusion. The avibactam ELF concentration was also somewhat nonlinear with respect to the plasma concentration, although to a lesser degree. This relationship was best described by a power model rather than a saturable model.

As ceftazidime and avibactam ELF:plasma ratios were higher at lower concentrations, ELF penetration was greater than previously calculated using non-compartmental AUC methods [16]. At ceftazidime plasma concentrations relevant for efficacy $(\sim 8 \mathrm{mg} / \mathrm{l})$, ELF penetration was $52 \%$, compared with an overall average across the time course of $\sim 32 \%$, as measured by AUC ratio, in the phase I study. Similarly, at plasma avibactam concentrations relevant for efficacy $(\sim 1 \mathrm{mg} / \mathrm{l})$, the corresponding penetration ratios were $47 \%$ in the present analysis versus $\sim 35 \%$ using non-compartmental methods. Thus, because of the non-linearity in ELF penetration of both drugs, simple AUC ratios can underestimate total ELF exposure, particularly since, at the later time points, which is around the efficacious concentration, ELF penetration is higher than at lower plasma concentrations. Moreover, the penetration ratios calculated here were relative to the total concentrations in plasma and ELF. Plasma free fraction in humans is estimated at $85 \%$ for ceftazidime and $92 \%$ for avibactam [28]. Protein levels in ELF are much lower than those in plasma [2] and, as such, the free fraction in ELF is likely to be higher than in plasma. Furthermore, both drugs demonstrate higher penetration in ELF in human models than in mouse models [13]. Therefore, calculation of penetration ratios (either using AUC ratios or at specific time points) using total plasma concentrations provides a conservative estimate of drug exposure in ELF.

Limitations of the analysis include use of the urea-correction method for determination of ELF fluid volumes from bronchoalveolar lavage samples, which may overestimate solute concentrations, and the lack of measurement of intracellular (alveolar macrophages) drug concentrations. While cellular uptake is minimal for the $\beta$-lactams [2], whether avibactam might penetrate into alveolar macrophages and thereby lead to overestimation of ELF concentrations (e.g. by cell lysis) is unknown. Moreover, interindividual variability in ELF antibiotic exposures can be high in critically ill patients [29], and the impact of pulmonary infection on ELF PK could not be determined from these healthy volunteer data. Nevertheless, penetration into ELF was substantial with both compounds. As no PK/PD targets based on ELF data were available, ELF concentrations were compared with mouse-derived plasma PK/PD targets [10-12]. Total ELF concentrations for simulated patients receiving the approved ceftazidime/avibactam dosage regimen (2000-500 mg q $8 \mathrm{~h}$ adjusted for renal function) were comparable to the plasma PK/PD targets for ceftazidime and avibactam, which are likely to be an overestimate of the ELF exposures required for efficacy. These findings are supported by results from the phase III REPROVE trial, in which ceftazidime/avibactam 2000-500 mg q8 h was shown to be non-inferior to meropenem $1 \mathrm{~g} \mathrm{q} 8 \mathrm{~h}$ (doses adjusted for renal function) with respect to clinical cure and all-cause mortality at day 28 [30, 31]. In REPROVE, clinical cure rates in patients with ceftazidime-non-susceptible pathogens were similar to those with ceftazidime-susceptible pathogens (29/36 [80.6\%] vs. $63 / 84$ [75.0\%], respectively), highlighting the efficacy of avibactam in this patient population [31]. It can therefore be inferred that patients with NP, including VAP, treated with ceftazidime/avibactam according to the approved dosage regimens achieve sufficient ceftazidime/avibactam exposures in the ELF for clinical efficacy.

In summary, the use of compartmental population PK methods such as those described here has broad applicability for the study of nonlinear drug penetration into the lung. Compared with simple AUC-based approaches, these methods may contribute to improving dose optimization of new antibiotics, and other drugs that target the lung.

Acknowledgements The authors thank all the subjects and investigators involved in the phase I trial of ceftazidime/avibactam penetration into ELF.

Funding This study was originally sponsored by AstraZeneca and is now sponsored by Pfizer. AstraZeneca's rights to ceftazidime/avibactam were acquired by Pfizer in December 2016. Editorial support was provided by Melanie More of Prime, Knutsford, Cheshire, UK, and funded by AstraZeneca and Pfizer.

Data sharing Upon request, and subject to certain criteria, conditions and exceptions (see https://www.pfizer.com/science/clinical-trials/trial -data-and-results for more information), Pfizer will provide access to individual de-identified participant data from Pfizer-sponsored global interventional clinical studies conducted for medicines, vaccines and medical devices (1) for indications that have been approved in the US and/or EU or (2) in programs that have been terminated (i.e., development for all indications has been discontinued). Pfizer will also consider requests for the protocol, data dictionary, and statistical analysis plan. Data may be requested from Pfizer trials 24 months after study completion. The de-identified participant data will be made available to researchers whose proposals meet the research criteria and other conditions, and for which an exception does not apply, via a secure portal. To gain access, data requestors must enter into a data access agreement with Pfizer.

\section{Compliance with Ethical Standards}

Conflict of interest Paul Newell and Shampa Das were employees of and shareholders in AstraZeneca at the time of this analysis. James G Wright is an employee of and shareholder in Wright Dose. Richard Dimelow and Merran MacPherson are former employees of Wright Dose. Merran MacPherson also holds shares in AstraZeneca. Wright Dose received funding from AstraZeneca for support and assistance with the population PK analysis.

Open Access This article is distributed under the terms of the Creative Commons Attribution-NonCommercial 4.0 International License (http://creativecommons.org/licenses/by-nc/4.0/), which permits any 
noncommercial use, distribution, and reproduction in any medium, provided you give appropriate credit to the original author(s) and the source, provide a link to the Creative Commons license, and indicate if changes were made.

\section{References}

1. Talbot GH. Considerations in undertaking a clinical development program for hospital-acquired bacterial pneumonia and/ or ventilator-associated bacterial pneumonia. Clin Infect Dis. 2010;51(Suppl 1):S144-9.

2. Kiem S, Schentag JJ. Interpretation of antibiotic concentration ratios measured in epithelial lining fluid. Antimicrob Agents Chemother. 2008;52(1):24-36.

3. Dallow J, Otterson LG, Huband MD, Krause KM, Nichols WW. Microbiological interaction studies between ceftazidime-avibactam and pulmonary surfactant and between ceftazidime-avibactam and antibacterial agents of other classes. Int J Antimicrob Agents. 2014;44(6):552-6.

4. Gonzalez D, Schmidt S, Derendorf H. Importance of relating efficacy measures to unbound drug concentrations for anti-infective agents. Clin Microbiol Rev. 2013;26(2):274-88.

5. Ambrose PG, Bhavnani SM, Ellis-Grosse EJ, Drusano GL. Pharmacokinetic-pharmacodynamic considerations in the design of hospital-acquired or ventilator-associated bacterial pneumonia studies: look before you leap! Clin Infect Dis. 2010;51(Suppl 1):S103-10.

6. Cazzola M, Blasi F, Terzano C, Matera MG, Marsico SA. Delivering antibacterials to the lungs: considerations for optimizing outcomes. Am J Resp Med Drugs Dev Other Interv. 2002;1(4):261-72.

7. Pfizer. Summary of Product Characteristics: Zavicefta. 2016. http://www.ema.europa.eu/docs/en_GB/document_library/ EPAR_-_Product_Information/human/004027/WC50021023 4.pdf. Accessed 1 May 2018.

8. Allergan. AVYCAZ (ceftazidime-avibactam) for injection, for intravenous use: prescribing information. Actavis, Inc. 2018. http://pi.actavis.com/data_stream.asp?product_group $=1957 \& p=$ pi\&language $=E$. Accessed 1 May 2018.

9. Ambrose PG, Bhavnani SM, Rubino CM, Louie A, Gumbo T, Forrest A, et al. Pharmacokinetics-pharmacodynamics of antimicrobial therapy: it's not just for mice anymore. Clin Infect Dis. 2007;44(1):79-86.

10. Coleman K, Levasseur P, Girard AM, Borgonovi M, Miossec $\mathrm{C}$, Merdjan $\mathrm{H}$, et al. Activities of ceftazidime and avibactam against beta-lactamase-producing Enterobacteriaceae in a hollowfiber pharmacodynamic model. Antimicrob Agents Chemother. 2014;58(6):3366-72.

11. Berkhout J, Melchers MJ, van Mil AC, Seyedmousavi S, Lagarde $\mathrm{CM}$, Schuck VJ, et al. Pharmacodynamics of ceftazidime and avibactam in neutropenic mice with thigh or lung infection. Antimicrob Agents Chemother. 2015;60(1):368-75.

12. Nichols WW, Newell P, Critchley IA, Riccobene T, Das S. Avibactam pharmacokinetic/pharmacodynamic targets. Antimicrob Agents Chemother. 2018;62(6):e02446-17.

13. Das S, Nichols WW, Li J. Dose selection of ceftazidime-avibactam in patients with nosocomial pneumonia, including ventilatorassociated pneumonia, based on preclinical efficacy, preclinical pharmacokinetic/pharmacodynamic and clinical pharmacokinetic data. In: Abstracts of the 25th European Congress of Clinical Microbiology and Infectious Diseases (ECCMID); 2015 25-28 April 2015; Copenhagen, Denmark, 2015. Abstract P1288.
14. Li J, Nichols WW, Zhou D, Das S. Population pharmacokinetic modeling of ceftazidime and avibactam and probability of target attainment to support the dosing regimen in patients with nosocomial pneumonia including ventilator-associated pneumonia. In: Abstracts of the 25th European Congress of Clinical Microbiology and Infectious Diseases (ECCMID); 2015; Copenhagen, Denmark, 2015. Abstract P12892015.

15. Li J, Zhou D, Das S, Lovern MR, Wada R, Bellanti F et al. PK/ $\mathrm{PD}$ target attainment analyses and assessment of dose adjustments for renal insufficiency for ceftazidime-avibactam (CAZ-AVI) in patients with complicated intra-abdominal infection (cIAI), complicated urinary tract infection (cUTI) or nosocomial pneumonia (NP). In: Abstracts of the American Association of Pharmaceutical Scientists (AAPS) Annual Meeting and Exposition; 2015 October 25, 2015; Orlando, FL, USA, 2015. Abstract 24592015.

16. Nicolau DP, Siew L, Armstrong J, Li J, Edeki T, Learoyd M, et al. Phase 1 study assessing the steady-state concentration of ceftazidime and avibactam in plasma and epithelial lining fluid following two dosing regimens. J Antimicrob Chemother. 2015;70(10):2862-9.

17. Andes D, Craig WA. Animal model pharmacokinetics and pharmacodynamics: a critical review. Int $\mathbf{J}$ Antimicrob Agents. 2002;19(4):261-8.

18. Andes D, Craig WA. Treatment of infections with ESBL-producing organisms: pharmacokinetic and pharmacodynamic considerations. Clin Microbiol Infect. 2005;11(Suppl 6):10-7.

19. MacVane SH, Kuti JL, Nicolau DP. Clinical pharmacodynamics of antipseudomonal cephalosporins in patients with ventilator-associated pneumonia. Antimicrob Agents Chemother. 2014;58(3):1359-64.

20. Muller AE, Punt N, Mouton JW. Optimal exposures of ceftazidime predict the probability of microbiological and clinical outcome in the treatment of nosocomial pneumonia. J Antimicrob Chemother. 2013;68(4):900-6.

21. Castanheira M, Farrell SE, Krause KM, Jones RN, Sader HS. Contemporary diversity of beta-lactamases among Enterobacteriaceae in the nine U.S. census regions and ceftazidime-avibactam activity tested against isolates producing the most prevalent beta-lactamase groups. Antimicrob Agents Chemother. 2014;58(2):833-8.

22. Flamm RK, Stone GG, Sader HS, Jones RN, Nichols WW. Avibactam reverts the ceftazidime MIC90 of European Gram-negative bacterial clinical isolates to the epidemiological cut-off value. J Chemother. 2014;26(6):333-8.

23. Levasseur P, Girard AM, Claudon M, Goossens H, Black MT, Coleman $\mathrm{K}$, et al. In vitro antibacterial activity of the ceftazidime-avibactam (NXL104) combination against Pseudomonas aeruginosa clinical isolates. Antimicrob Agents Chemother. 2012;56(3):1606-8.

24. Nichols WW, de Jonge BL, Kazmierczak KM, Karlowsky JA, Sahm DF. In vitro susceptibility of global surveillance isolates of Pseudomonas aeruginosa to Ceftazidime-Avibactam (INFORM 2012 to 2014). Antimicrob Agents Chemother. 2016;60(8):4743-9.

25. Clinical Laboratory Standards Institute. Performance standards for antimicrobial susceptibility testing. Twenty-eighth informational supplement. Wayne: CLSI; 2018.

26. Das S, Riccobene TA, Carrothers TJ, Wright J, Macpherson M, Lovern MR et al. Pharmacokinetic/pharmacodynamic validation of the ceftazidime-avibactam dose in patients with nosocomial pneumonia, including ventilator-associated pneumonia. In: Abstracts of the 27th European congress of clinical microbiology and infectious diseases (ECCMID); 2017 22-25 April 2017; Vienna, 2017.

27. Rodvold KA, George JM, Yoo L. Penetration of anti-infective agents into pulmonary epithelial lining fluid: focus on antibacterial agents. Clin Pharmacokinet. 2011;50(10):637-64. 
28. Crandon JL, Schuck VJ, Banevicius MA, Beaudoin ME, Nichols WW, Tanudra MA, et al. Comparative in vitro and in vivo efficacies of human simulated doses of ceftazidime and ceftazidimeavibactam against Pseudomonas aeruginosa. Antimicrob Agents Chemother. 2012;56(12):6137-46.

29. Felton TW, McCalman K, Malagon I, Isalska B, Whalley S, Goodwin J, et al. Pulmonary penetration of piperacillin and tazobactam in critically ill patients. Clin Pharmacol Ther. 2014;96(4):438-48.

30. Torres A, Rank D, Rekeda L, Chen X, Riccobene TA, Critchley I, et al. Phase 3, randomized, double-blind noninferiority study of ceftazidime-avibactam vs meropenem in the treatment of patients with hospital-acquired bacterial pneumonia and ventilator-associated bacterial pneumonia: analyses of the REPROVE study per FDA endpoints. Open Forum Infect Dis. 2017;4(Suppl 1):S535.

31. Torres A, Zhong N, Pachl J, Timsit JF, Kollef M, Chen Z et al. Ceftazidime-avibactam versus meropenem in nosocomial pneumonia, including ventilator-associated pneumonia (REPROVE): a randomised, double-blind, phase 3 non-inferiority trial. Lancet Infect Dis. 2018;18(3):285-95. 